EPJ Web of Conferences 53, 01013 (2013)

DOI: $10.1051 /$ epjconf/20135301013

(C) Owned by the authors, published by EDP Sciences, 2013

\title{
Interpretation of ultra-high energy multi-messenger data
}

\author{
Günter Sigla \\ II. Institut für theoretische Physik, Universität Hamburg, Luruper Chaussee 149, \\ 22761 Hamburg, Germany
}

\begin{abstract}
Charged ultra-high energy cosmic rays above $\sim 10^{17} \mathrm{eV}$ are most likely accelerated in powerful astrophysical objects that have to meet a number of requirements to reach energies beyond the highest energies observed, a few $10^{20} \mathrm{eV}$, among them sufficient electromagnetic power and further constraints on geometry and magnetic fields to avoid excessive energy losses during acceleration. Since charged particles are deflected in cosmic magnetic fields and do not always point back to their sources, it is challenging to identify them and to reconstruct a detailed understanding of the acceleration mechanism. Neutral secondaries such as $\gamma$-rays and neutrinos that are produced by interactions of primary cosmic rays within the source and during propagation can provide important complementary information. We provide a brief overview over how multi-messenger data can be exploited to learn more about source and propagation physics.
\end{abstract}

\section{INTRODUCTION}

In this paper we will discuss some aspects of the interplay between high energy charged cosmic rays, $\gamma$-rays and neutrinos, a topic that is often called "high energy multi-messenger astrophysics". Only primary charged cosmic rays can be accelerated in electromagnetic fields whereas secondary photons and neutrinos have to be produced by interactions either within the sources or during propagation from the source to Earth. Concerning the primary cosmic rays we will focus here on ultra-high energy cosmic rays (UHECRs) which here are understood to be cosmic rays above $10^{17} \mathrm{eV}$. Since secondary neutral particles tend to have lower energies due to the kinematics of their production and (in case of photons) electromagnetic cascading, neutrinos down to $\mathrm{PeV}\left(=10^{15} \mathrm{eV}\right)$ and $\gamma$-rays down to $\mathrm{GeV}$ energies will be relevant to our discussion. Even radio astronomy can play a role in multi-messenger astrophysics since for example luminous radio galaxies are considered to be promising UHECR sources.

In Sect. 2 we briefly review the physics of cosmic ray acceleration. Nearby source candidates such as the radio galaxy Centaurus $\mathrm{A}$ have been observed in photons from radio frequencies to $\mathrm{TeV}$ energies and we briefly discuss the modeling efforts of their fluxes in the context of cosmic ray acceleration. Neutrinos and $\gamma$-rays are often produced through pion production implying a close connection between the two as well as to the primary cosmic ray flux. This will be discussed in Sect. 3 for diffuse UHE $\gamma$-rays and neutrinos produced by interactions during propagation and for the expected neutrino fluxes from Gamma Ray Bursts. In Sect. 4 we briefly discuss the effects of galactic and extragalactic magnetic fields on the time structure of UHECR fluxes from particular sources and on anisotropies of UHECR fluxes. In Sect. 5 we show how one can use UHE photons produced by pion production of primary UHECRs to constrain tiny violations of Lorentz symmetry.

We use natural units, $c_{0}=\hbar=1$, throughout.

\footnotetext{
${ }^{\mathrm{a} e}$-mail: guenter.sigl@desy.de
}

This is an Open Access article distributed under the terms of the Creative Commons Attribution License 2.0, which permits unrestricted use, distribution, and reproduction in any medium, provided the original work is properly cited. 


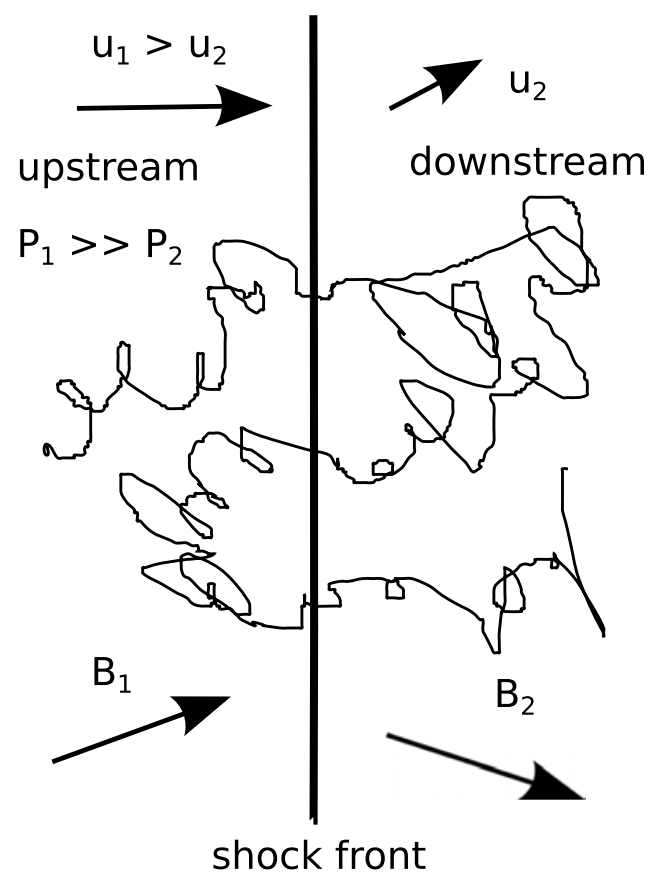

Figure 1. For a plane, adiabatic shock the jumps in mass density, velocity, and pressure are given by the RankineHugoniot conditions and determine the compression ratio $r \equiv \rho_{2} / \rho_{1}$. Upstream and downstream regions are denoted with index 1 and 2, respectively.

\section{SOURCES OF ULTRA-HIGH ENERGY COSMIC RAYS}

Astrophysical media are usually partially or fully ionized and highly conductive. Therefore, any electric field would induce large electric currents which would rearrange charges such that the electric fields are screened. As a consequence, an astrophysical plasma cannot carry any significant electric field in its rest frame in an equilibrium situation and only magnetic fields are allowed which often have a complicated structure. However, since the energy of a charged particle is conserved in a magnetic field, such particles can not be accelerated in uniformly moving plasmas.

The situation changes in a system consisting of at least two plasma frames moving with respect to each other. Where they meet a shock is formed which basically is a solution of the hydrodynamics equations with a discontinuity in the flow of a fluid or plasma. We will here only consider plane shocks. In the "shock frame" in which the shock is at rest, the plasma is moving toward the shock with a velocity $u_{1}$ from the "upstream" side, and moving away from the shock on the opposite, "downstream" side with a velocity $u_{2}<u_{1}$. The situation is depicted in Fig. 1 where we define the positive $z$-direction as perpendicular to the shock front pointing toward the downstream region. In the Fermi acceleration process, cosmic rays cross back and forth across astrophysical shocks by scattering on inhomogeneities of the magnetic fields. As we will show in the following, in each shock crossing, on average they gain energy, but also have a finite probability for escaping the acceleration region. This interplay between energy gain and particle loss will lead to a power law spectrum.

We will not enter into the details of shock acceleration theory here. We only mention that the diffusion coefficient in the magnetic field is limited by Bohm diffusion,

$$
D(p) \gtrsim \frac{1}{3} r_{g}(p) \simeq \frac{p}{e Z B_{\perp}},
$$


where $r_{g}(p)$ is the gyro radius of a particle of charge $e Z$ and momentum $p$ in a magnetic field $B$. The acceleration time scale can then be estimated as

$$
T_{\mathrm{acc}}(p) \simeq \frac{3 D(p)}{8\left(u_{1}-u_{2}\right) u_{2}}
$$

The situation described above can then lead to power laws of slope around $d N / d e \propto E^{-2}$ for strong shockes. For comprehensive reviews of shock acceleration theory see, for example, Ref. [1, 2]. We are mostly interested in the maximal energy up to which cosmic rays can be accelerated in shocks. In this section we consider all quantities in the shock rest frame. If the shock itself moves with a Lorentz factor $\Gamma$, there will be a geometry-dependent relation between quantities in the shock rest frame and in the observer frame.

At very high energies, the escape time from the shock is given by the diffusion time over the finite linear size $R$ of the shock,

$$
T_{\mathrm{esc}}^{R} \simeq \frac{R^{2}}{D(p)} \gtrsim \frac{R^{2}}{r_{g}(p)} \simeq \frac{e Z B_{\mathrm{rms}} R^{2}}{p},
$$

where we have used Eq. (1). Since acceleration requires $T_{\text {acc }} \lesssim T_{\text {esc }}^{R}$, using Eq. (2) for the acceleration time scale with $D(p) \gtrsim r_{g}(p)$, this results in the famous Hillas criterion [3],

$$
E_{\max } \lesssim e Z R B v \simeq 10^{18} v\left(\frac{B}{\mu \mathrm{G}}\right)\left(\frac{R}{\mathrm{kpc}}\right) \mathrm{eV},
$$

where we have abbreviated the root mean square of the magnetic field $B_{\mathrm{rms}}$ by $B$. There is another, quicker and more qualitative derivation of Eq. (4) that also gives an order of magnitude estimate of the acceleration time scale Eq. (2): The time scale at which diffusion and convection length scales become comparable in the shock, $d \sim D(p) t \sim(v t)^{2}$, is given by $t \sim D(p) / v^{2}$. Requiring that the corresponding length scale $d$ is smaller than the shock size, $d \simeq D(p) / v \lesssim R$ immediately results in Eq. (4) when Eq. (1) is used.

Note that for relativistic shocks, $v \simeq 1$, the Hillas criterion is equivalent to the intuitive condition that the gyro radius has to be smaller than the size of the shock, $r_{g}(p) \lesssim R$. The Hillas criterion is shown and compared with various astrophysical objects in Fig. 2.

Accelerating particles of charge $e Z$ to an energy $E_{\max }$ also requires a minimal source power which can be estimated as follows: Acceleration to an energy $E_{\max }$ requires an induction $\mathcal{E} \gtrsim E_{\max } /(e Z)$. With $Z_{0} \simeq 100 \Omega$ the vacuum impedance, this leads to the dissipation of a minimal power of $[4,5]$

$$
L_{\min } \simeq \frac{\mathcal{E}^{2}}{Z_{0}} \simeq 10^{45} Z^{-2}\left(\frac{E_{\max }}{10^{20} \mathrm{eV}}\right)^{2} \mathrm{erg} \mathrm{s}^{-1}
$$

When expressing the square of the product of the magnetic field in an accelerator with its size in terms of a luminosity, $L \sim B^{2} R^{2}$, this condition can be expressed in terms of the Hillas-criterium Eq. (4) for relativistic shocks, $v \simeq 1$, which states that the gyro radius of a charged particle at the maximal acceleration energy must fit within the accelerator of size $R$. Eq. (5) suggests that the power requirements are considerably relaxed for heavier nuclei which is easy to understand because an estimate solely based on motion of charged particles in magnetic fields can only depend on their rigidity $E / Z$.

However, the Hillas criterion Eq. (4) and the minimal power Eq. (5) are necessary but in general not sufficient since they do not take into account energy loss processes within the source. If the approximation of collision-less acceleration is not good, the maximal energy is further constrained by the condition that the acceleration time scale must not only be smaller than the escape time, but also smaller than the energy loss time over which the particle looses a given fraction of its energy. The most important energy loss processes for cosmic rays are pion production, photo-disintegration in case of nuclei, Bethet-Heitler pair production and, in case of very compact accelerators and/or high magnetic fields, synchrotron emission and curvature radiation. Furthermore, the shock may only have a finite 


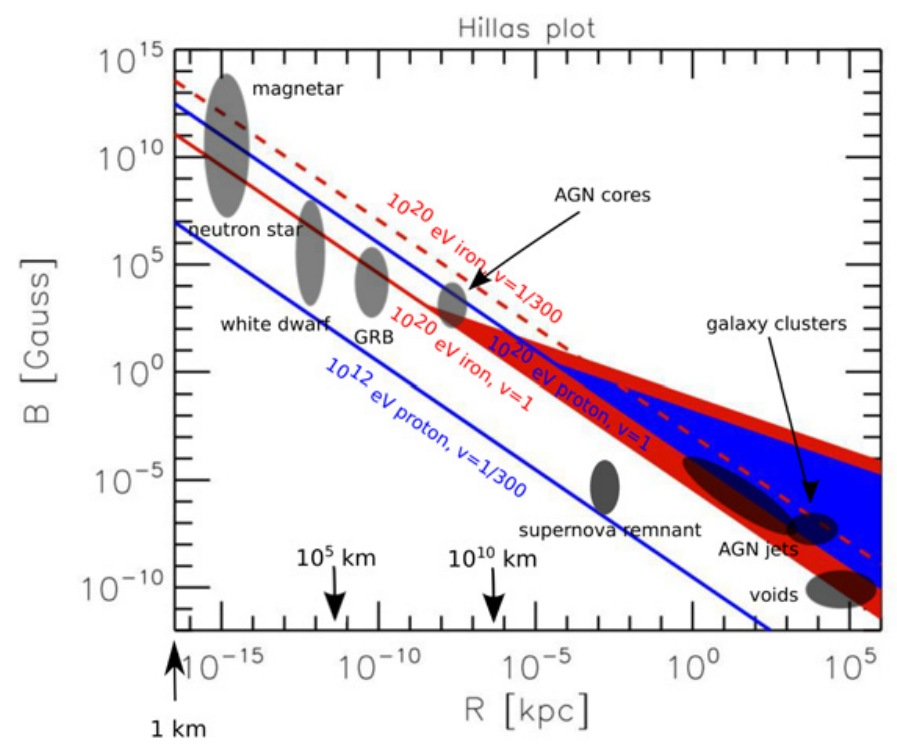

Figure 2. The "Hillas plot" represents astrophysical objects which are potential cosmic-ray accelerators on a twodimensional diagram where on the horizontal direction the size linear extension $R$ of the accelerator, and on the vertical direction the magnetic field strength $B$ are plotted. According to Eq. (4), the maximal acceleration energy $E$ is proportional to $Z R B v$, where $v$ is the shock velocity in units of the speed of light and $Z$ is the particle charge. Particular values for the maximal energy correspond to diagonal lines in this diagram and can be realized either in a large, low field acceleration region or in a compact accelerator with high magnetic fields. For a shock velocity $v \sim 1$, neutron stars, AGN, Radio Galaxies or Galactic clusters can accelerate protons to $E \sim 10^{20} \mathrm{eV}$. For typical nonrelativistic shocks, $v \sim 1 / 300$, as they are realized, for example, in supernova remnants, no astrophysical objects of sufficient size and magnetic field to produce $10^{20} \mathrm{eV}$ protons are known. The blue and red shaded wedges signify the parameter ranges satisfying both the Hillas condition Eq. (4) and the synchrotron condition Eq. (7) for a $10^{20} \mathrm{eV}$ proton and iron, respectively in the shock rest frame.

lifetime $T_{\mathrm{dyn}}$ which gives an additional condition. The most general version of the equation determining the maximal energy is thus

$$
T_{\mathrm{acc}}(p) \lesssim \min \left[T_{\mathrm{esc}}^{L}(p), T_{\mathrm{loss}}(p), T_{\mathrm{dyn}}\right] .
$$

The modifications resulting form taking into account energy loss processes have recently been discussed in Ref. [6]. For example, for diffuse shock acceleration synchrotron radiation by the accelerated nuclei is the dominant energy loss mechanism and yields the additional constraint

$$
E_{\max } \lesssim 3 \times 10^{16} \frac{A^{4}}{Z^{4}}\left(\frac{B}{\mathrm{G}}\right)^{-2}\left(\frac{R}{\mathrm{kpc}}\right)^{-1} \mathrm{eV} .
$$

This equation essentially follows from integrating the synchrotron energy loss rate

$$
\left.\frac{d E}{d t}\right|_{\mathrm{syn}} \simeq-\frac{e^{4}}{36 \pi^{2} m_{p}^{4}}\left(\frac{Z}{A}\right)^{4} B^{2} E^{2}
$$

over the length scale $R$ [7], where $m_{p}$ is the proton mass. The additional constraint Eq. (7) is shown for $10^{20} \mathrm{eV}$ protons and iron nuclei as the colored wedges in Fig. 2.

In terms of these constraints the most promising sources for the highest energy cosmic rays are still considered to be active galactic nuclei (AGNs) and Gamma Ray Bursts (GRBs). Both are time variable as the AGN activity is thought to be due to an intermittent accretion history of stars and gas onto a supermassive central black hole and GRBs are known to occur through the merger of binary neutron 


\section{UHECR 2012}

stars or the core collapse of a massive star on times scales of tens of seconds. This variability is visible in photons and should be visible in neutrinos, once some are detected from these sources. In contrast, time delay by deflection in cosmic magnetic fields strongly modifies the time structure of the charged UHECRs as we will briefly see in Sect. 4.

Acceleration is often assumed to be rigidity limited such that the differential flux of nuclei of charge $Z$ at energy $E$ can, for example, be assumed to be of power-law form

$$
\Phi_{Z, A}(E)=\frac{d N_{Z, A}}{d E} \propto q_{Z, A} E^{-\alpha} \Theta\left(Z E_{\max , \mathrm{p}}-E\right),
$$

where $q_{Z, A}$ is the relative abundance of the nucleus of charge $Z$ and atomic number $A$ at a given energy $E, \alpha$ is the differential spectral index, $E_{\max , \mathrm{p}}$ is the maximal proton energy and $\Theta(x)$ is the Theta-function which cuts off the spectrum for $E>Z E_{\max , \mathrm{p}}$. To compare with abundances in a non-relativistic gas, one often refers to abundances $x_{Z, A}$ at a given energy per nucleon $E / A$, such that for a differential spectrum with power-law index $s$ one has

$$
q_{Z, A} \propto x_{A, Z} A^{\alpha-1} .
$$

Thus, a steep spectrum can lead to a strong enhancement of the abundance of heavy nuclei compared to the abundances in the gas at rest.

The Pierre Auger data shows a clustering of super-GZK events towards the direction of the closest known radio galaxy, Centaurus A (NGC 5128, "Cen A") [8]. Two events even correlate with the nucleus of this galaxy. This may be somewhat surprising since, although Cen A is the closest radio galaxy at a distance of just $\simeq 3.6 \mathrm{Mpc}$ and the third-strongest radio source in the sky, it is an elliptical radio galaxy with a relatively small power output [13] which makes it difficult to reach the required UHECR energies. On the other hand, UHECR events observed towards Cen A could originate mainly from sources within the Centaurus galaxy cluster which is itself part of the Hydra-Centaurus supercluster and located just behind Cen A. In any case, Cen A has been observed in many channels and its small distance allows detailed astronomical and astrophysical studies. For example, its lobes have been detected in $200 \mathrm{MeV}$ $\gamma$-rays by Fermi Large Area Telescope (Fermi LAT) [9]. For a magnetic field of $\simeq 0.85 \mu \mathrm{G}$ the low energy peak can be explained as synchrotron emission by relativistic electrons whereas the high energy peak would be due to inverse Compton scattering of the same electrons on the cosmic microwave background (CMB). In this model the power radiated in synchrotron and inverse Compton scattering is proportional to the energy densities in the magnetic field and the low energy ambient photon field, respectively. Since the latter is dominated by the CMB whose energy density is well known, the magnetic field strength can be determined from the observed power ratio of the two peaks. The core of Cen A was also observed by Fermi LAT [10] and again shows two humps which can be explained by synchrotron emission of accelerated electrons and inverse Compton scattering of the same electrons, this time on the very synchrotron photons they radiated ("synchrotron-self Compton model"). Cen A has also been observed in $\mathrm{TeV} \gamma$-rays by the H.E.S.S. telescopes [11]. This component, however, cannot be explained by the electrons that presumably the two lower energy peaks. The $\mathrm{TeV} \gamma$-rays may then be due to pions produced by the interactions of protons accelerated in Cen A [12]. These same primary cosmic rays may then extend to sufficiently high energies to give rise to the UHECR flux excess observed by the Pierre Auger Observatory [8].

Multi-wavelength and multi-messenger observations of Cen A and its potential role as a major local UHECR accelerator has triggered many multi-messenger model building efforts for Cen A [13, 14]. For example, it has been pointed out in Ref. [14] that proton acceleration in the jet of Cen A is hard to reconcile with observations of $\mathrm{Cen} \mathrm{A}$ in $\mathrm{TeV}$ gamma-rays by the H.E.S.S. telescopes [11] if gamma-rays are produced by proton-proton interactions. Instead, $\mathrm{p}-\gamma$ interactions in the core are more consistent with these observations.

The composition of UHECRs accelerated in specific sources can even have signatures in $\mathrm{GeV}$ $\gamma$-rays, as has recently been pointed out in Ref. [15]: Nuclei leaving the sources are usually fully 
ionized due to the strong radiation fields within the sources. Some of these nuclei are unstable and undergo beta-decay with the produced electron being captured by the nucleus and thus forming an ion with a single bound electron. A cycle of excitation and de-excitation of this one-electron ion can then lead to a $\gamma$-ray flux that peaks around $8 \mathrm{GeV}$ for iron. Other nuclei would lead to different peak energies so that an observation of these features could even contain information on the nuclear composition of the source. The $\gamma$-ray flux observed from Cen A, for example, is so far consistent with a substantial iron component accelerated to $\sim 10^{17} \mathrm{eV}$ in this source [15].

An interesting argument linking UHECR sources to their luminosity at radio frequencies has been put forward in this context by Hardcastle [16]. The lobes of radio galaxies accelerating cosmic rays of charge $Z$ to an energy $E$ should have a minimal luminosity at radio frequencies around $108 \mathrm{MHz}$ given by

$$
L_{108 \mathrm{MHz}} \gtrsim 2 \times 10^{24} \epsilon\left(\frac{E / Z}{10^{20} \mathrm{eV}}\right)^{7 / 2}\left(\frac{r_{\text {lobe }}}{100 \mathrm{kpc}}\right)^{-1 / 2} \mathrm{WHz}^{-1},
$$

where $r_{\text {lobe }}$ is the lobe radius and $\epsilon$ is the energy in electrons relative to the energy in the magnetic field. The scalings in Eq. (11) can be understood as follows: An electron population with spectral density per volume $d n_{e} / d E=N_{0} E^{-s}$ in a magnetic field of strength $B$ at a given frequency $v$ emits the power per frequency and volume scaling as $(d P / d v)(v) \propto N_{0} B^{(s+1) / 2} v^{(1-s) / 2}$. By definition of $\epsilon$ one has $N_{0} \propto B^{2}$ which can be used to eliminate the normalization $N_{0}$. Finally, using the Hillas condition Eq. (4) gives $B \gtrsim E /(e Z R)$. Putting everything together one obtains for the total source power per frequency $L_{v} \propto R^{3}(d P / d v)(v)$ at frequency $v$ a lower limit that scales as $L_{v} \propto \epsilon(E / Z)^{(s+5) / 2} R^{(1-s) / 2} v^{(1-s) / 2}$. For a canonical power law index $s=2$ this results in the scaling of Eq. (11) if one identifies $R$ with the lobe radius $r_{\text {lobe. }}$ Ref. [16] concludes from these estimates that if UHECRs are predominantly protons, then very few sources should contribute to the observed flux. These sources should be easy to identify in the radio and their UHECR spectrum should cut off steeply at the observed highest energies. In contrast, if the composition is heavy at the highest energies then many radio galaxies could contribute to the UHECR flux because the predicted power at radio frequencies, Eq. (11), would be reduced by a large power of $Z$. In this case, due to the much stronger deflection, only the nearby radio galaxy Centaurus $\mathrm{A}$ may be identifiable as a UHECR source.

\section{RELATIONS BETWEEN GAMMA-RAY, NEUTRINO AND PRIMARY COSMIC RAY FLUXES}

$\gamma$-rays are produced by decaying neutral pions created by photo-hadronic interactions of primary cosmic rays or by inverse Compton scattering of electrons and positrons that are part of an electromagnetic cascade or are produced by Bethe-Heitler pair production by charged primary cosmic rays. In Sect. 2 we already discussed the role of $\gamma$-rays for the understanding of UHECR sources. Here, we consider the case where a continuous distribution of sources injects a mixed composition of UHE nuclei up to a redshift of $z=2$ with an injection rate per comoving volume increasing proportional to $(1+z)^{4}$, slightly more strongly than motivated by the star formation history.

During their propagation from the source to the observer these nuclei produce secondary neutrinos and $\gamma$-rays, the latter of which induce electromagnetic cascades on the CMB and infrared backgrounds. The resulting primary UHECR fluxes can be fitted to the data and the secondary $\gamma$-ray and neutrino fluxes can be compared to existing upper limits, as is shown in Fig. 3. These fluxes have been simulated with CRPropa 2.0 [17]. Note that the diffuse $\gamma$-ray background observed by Fermi LAT at $10-100 \mathrm{GeV}$ energies [26] is not far above the predictions in this scenario and can become a serious constraint of such scenarios with an steadily improving understanding of this diffuse $\gamma$-ray background. We also stress that the shape of the spectra is quite different because neutrinos do not interact once produced and thus reflect the spectrum at production whereas $\gamma$-rays undergo an electromagnetic cascade which is an alternating cycle of pair production and inverse Compton scattering which shifts most of the energy 


\section{UHECR 2012}

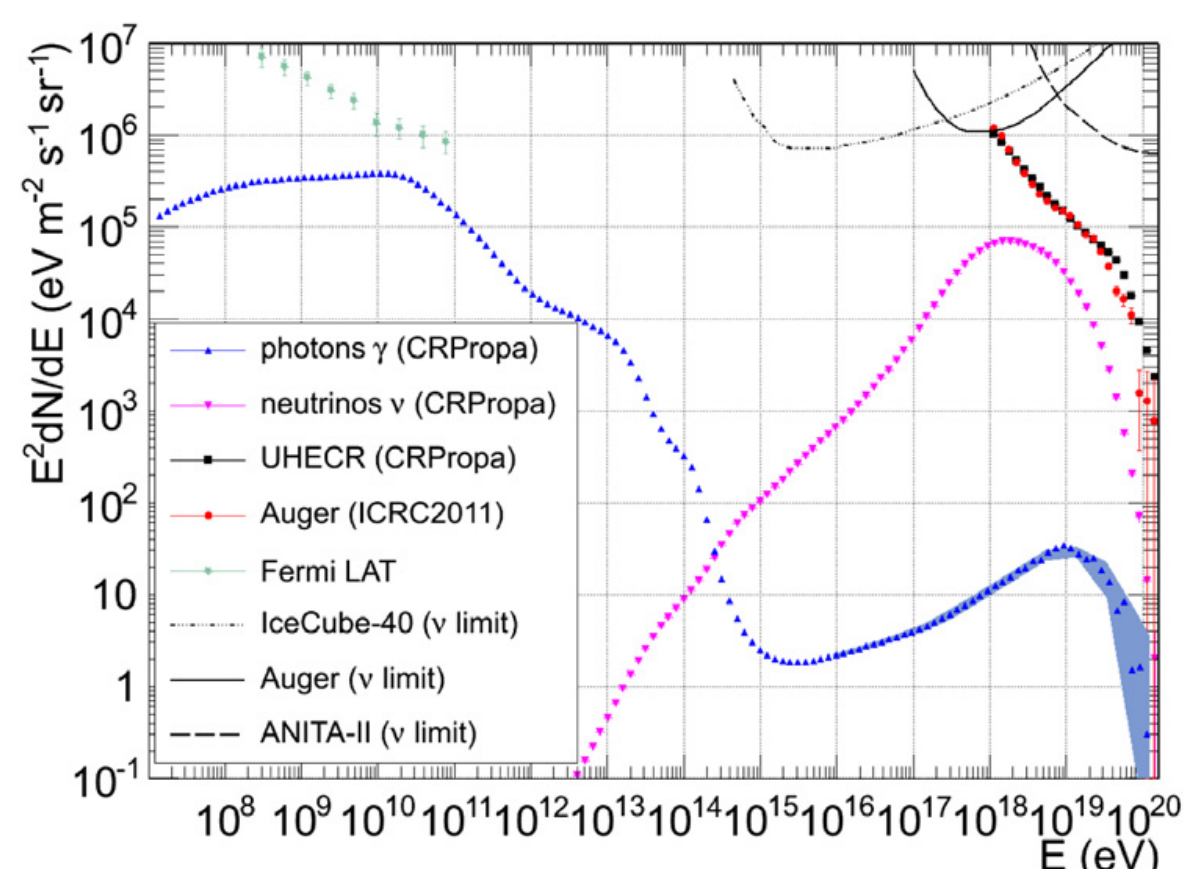

Figure 3. All-sky fluxes of primary UHECRs (black rectangles), spectra of secondary $\gamma$-rays (blue triangles) and neutrinos (magenta triangles) predicted by a continuous distribution of sources injecting a mixed composition of UHE nuclei following a galactic mass distribution $[18,19]$ with a spectrum given by Eq. (9) with maximal rigidity $E_{\max , \mathrm{p}}=384.6 \mathrm{EeV}$ and $\alpha=2.2$ up to a redshift of $z=2$ with an injection rate per comoving volume increasing proportional to $(1+z)^{4}$. The primary UHECR flux has been normalized to the Pierre Auger spectrum (red dots) [20,21]. The neutrino flux shown is the flux per flavor, assuming maximal flavor mixing with the fluxes of all flavors being equal. For comparison shown are the single-flavor neutrino flux limits (black lines) [22-24]. The $\gamma$-ray flux above $10^{18} \mathrm{eV}$ is consistent with the Pierre Auger limits [25]. Green stars show the isotropic $\gamma$-ray flux measured by Fermi-LAT [26]. Figure taken from Ref. [17].

below the threshold for pair production on the CMB around $10^{15} \mathrm{eV}$. Despite that, the energy fluence in $\gamma$-rays and neutrinos which is proportional to the area below the respective flux curve in Fig. 3 is roughly equal for $\gamma$-rays and neutrinos because a comparable amount of energy goes into $\gamma$-rays and neutrinos due to the approximate isospin symmetry in pion production.

It is clear that the fluxes of $\gamma$-rays and neutrinos produced during propagation essentially depend on the injected UHECR flux at energy per nucleon above the GZK threshold. As a result, these neutral secondary fluxes will strongly depend on the UHECR source properties, in particular the mass composition and the maximal energy. The neutrino flux which is not absorbed during propagation will in addition strongly depend on the redshift evolution of the sources [27]. This is not the case for the secondary UHE photon flux which is subject to pair production on the CMB and radio backgrounds within a few Mpc. There is thus a certain amount of complementarity in the information contained in secondary UHE photons and neutrinos which can provide indirect information on the source properties once photons and/or neutrinos above $\sim 10^{17} \mathrm{eV}$ will be detected. Even improved upper limits will allow to constrain at least some of the more extreme scenarios such as proton dominated fluxes with very high maximal energies and strong redshift evolution.

Electromagnetic cascades such as the one shown in Fig. 3 for the case of diffuse fluxes also play a role for the observation of distant blazars in $\mathrm{GeV}$ and $\mathrm{TeV} \gamma$-rays. The latter should be strongly absorbed by pair production in the infrared background above a redshift dependent energy which is a few $\mathrm{TeV}$ at $z \simeq 0.1$ and drops to $\simeq 0.3 \mathrm{TeV}$ above a redshift $z \simeq 0.5$ [28]. Despite this prediction the 
$\mathrm{TeV} \gamma$-ray fluxes of some distant blazars at redshifts up to $z \simeq 0.5$ seem to be less attenuated than expected if the $\gamma$-rays are directly produced within the source. This has variously been named as a "pair production anomaly" [28]. It has been suggested that if the same sources also emit UHECRs the continuous production of secondary $\gamma$-rays along the line of sight can harden the TeV $\gamma$-ray spectrum and thus avoid the pair production anomaly [29]. At the same time some of these blazars are not observed in $\mathrm{GeV} \gamma$-rays by the Fermi LAT experiment although a straightforward extrapolation of their $\mathrm{TeV}$ fluxes would suggest they should be easily visible in $\mathrm{GeV} \gamma$-rays. This can be explained if the electromagnetic cascade is dispersed by intergalactic magnetic field on angles larger than the field of view of Fermi LAT [30]. Overall these two effects would constrain magnetic fields in the large scale voids traversed by the electromagnetic cascades to be in the range between $\sim 10^{-17} \mathrm{eV}$ and $\sim 10^{-14} \mathrm{eV}$ [31]. We will not go into further details of these blazar observations but instead refer to the contribution of Alexander Kusenko in the same conference.

The situation in high energy extraterrestrial neutrino astrophysics at PeV energies is currently very interesting: One the one hand the IceCube experiment has put upper limits on the neutrino flux from individual Gamma Ray Bursts (GRBs) [32, 33] as well as on the diffuse neutrino flux above $10^{13} \mathrm{eV} \mathrm{[34].}$ On the other hand, IceCube has recently reported two candidate neutrino events with energies around a $\mathrm{PeV}$, but with so far unknown arrival directions [35]. Are these neutrinos from UHECR sources such as AGNs or GRBs? We don't know yet but the flux due to these two candidate events is consistent with the current upper limits from IceCube. We will, therefore, focus on the implications of these upper limits for now which start to strongly constrain fireball models for GRBs and their predictions of associated neutrino fluxes. Note that in contrast to the cosmogenic or GZK neutrinos an example of which were given in Fig. 3 and which are produced during propagation, these neutrinos are produced within the GRB source and have lower energies around PeV energies. Their fluxes can be estimated in several ways:

In scenarios in which the sources of UHECR are primarily GRBs [36, 37] the neutrino flux can be linked to the observed UHECR flux. In these scenarios the neutrons escaping from the GRB whose $\beta-$ decay back into protons which give rise to the UHECR flux $\Phi_{p}(E)$ per energy from the GRB. Therefore, the neutrino per energy flux from the interaction of the original accelerated proton, $p \gamma \rightarrow n \pi^{+}$, can be directly related to $\Phi_{p}(E)$ via

$$
\Phi_{\nu}\left(E_{\nu}\right) \sim \frac{1}{\eta_{\nu}} \Phi_{p}\left(\frac{E}{\eta_{\nu}}\right),
$$

where $\eta_{v} \simeq 0.1$ is the average neutrino energy in units of the parent proton energy. This applies as long as the pions and muons decay faster than they loose energy by interactions within the GRB. Above a certain critical energy $E_{\pi, \mu}^{c}$ which is typically of the order of $10^{17} \mathrm{eV}$, this is not the case any more because of the prolonged pion and muon lifetimes due to time dilation and the neutrino flux above that energy is suppressed by a factor $\simeq E_{\pi, \mu}^{c} /\left(4 E_{v}\right)$ compared to Eq. (12) which reflects the probability that the pion or muon decays before interacting. Note that the contribution of GRBs to UHECRs is itself uncertain since it is proportional to the baryon loading factor $f_{b}$ which is basically the ratio of the total energy in protons to the total energy in electrons. The diffuse high energy neutrino flux is then obtained by folding Eq. (12) with the cosmological GRB rate and integrating over redshift. In general, the resulting flux predictions are higher than current flux limits, as has been shown for the neutrino flux associated with the prompt phase in fireball scenarios in which associated UHECR fluxes are matched with observations, for example, in Ref. [38]. This does, however, not rule out scenarios in which most of the UHECRs are produced by GRBs, because the amount of energy transferred from protons accelerated in the GRBs is uncertain by at least factors of a few [39]. A very detailed discussion of such uncertainties has recently given in the review Ref. [40].

Alternatively, GRBs may not significantly contribute to the UHECR flux. In this case one can predict neutrino energy fluences from the observed GRB photon fluences [32] via simplified analytical formuli 


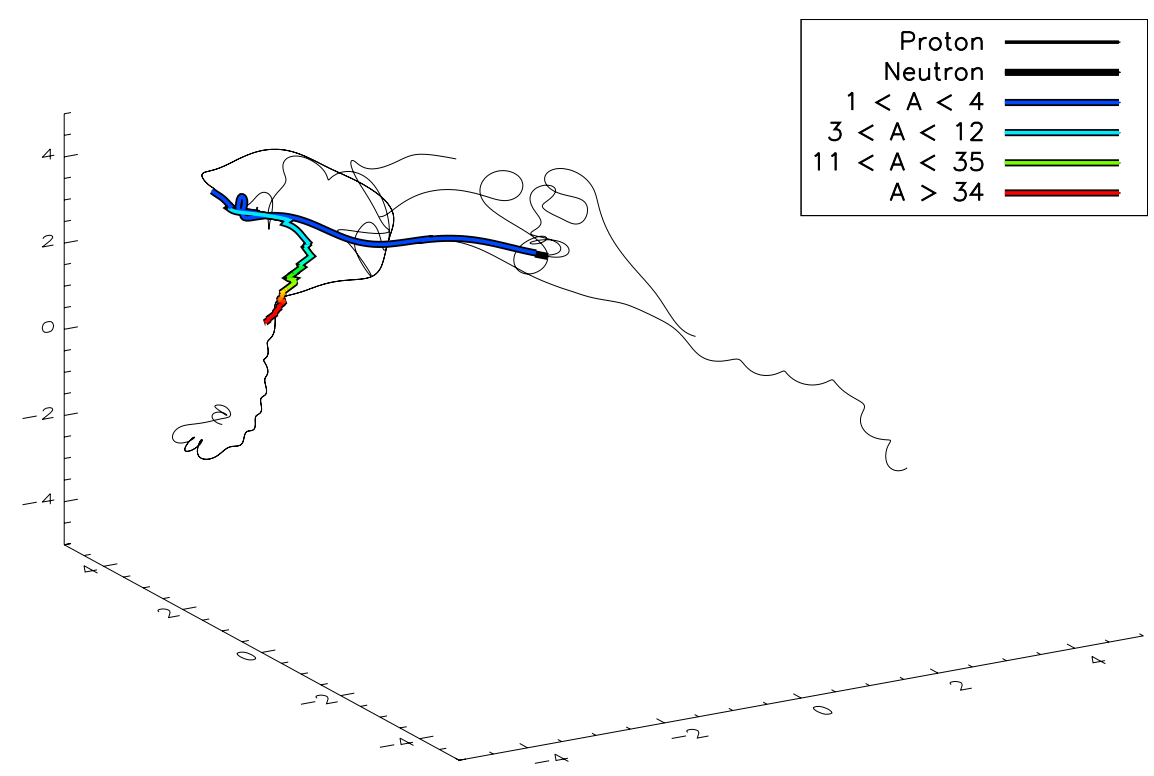

Figure 4. A typical trajectory initiated by an iron nucleus at an initial energy of $10^{21} \mathrm{eV}$ in a structured EGMF.

such as

$$
\int_{0}^{\infty} d E_{\nu} E_{v} \frac{d N_{v}}{d E_{v}} \sim f_{b} f_{\pi} \int_{1 \mathrm{keV}}^{10 \mathrm{MeV}} d E_{\gamma} E_{\gamma} \frac{d N_{\gamma}}{d E_{\gamma}},
$$

where $f_{\pi}$ is the pion production efficiency of the protons. The neutrino fluxes can also be calculated in much more detail numerically by taking into account the detailed proton and photon energy distributions (which are deduced from the observed GRB photon spectra) as well as the detailed cross section energy dependencies including multi-pion and kaon production and cooling of secondary particles. In these kind of simulations the predicted neutrino fluxes turn out to be about an order of magnitude smaller [41, 42] compared to the simplified analytical estimates. This suggests that the IceCube observations are not yet in severe tension with GRB models.

\section{COSMIC MAGNETIC FIELDS AND THREE-DIMENSIONAL PROPAGATION}

In this section we briefly consider effects of large scale magnetic fields on UHECR propagation, in particular on sources which do not produce UHECRs continuously but either in brief bursts, such as for GRBs and AGN flares [43, 44], or over longer episodes such as AGNs which have a duty cycle of about $1 \%$ in their $\mathrm{X}$-ay emission.

The propagation of three-dimensional UHECR trajectories has to be studied numerically. Several numerical codes have recently been developed, including HERMES, SimProp and CRPropa 2.0. We will here focus on CRPropa since it has recently been released to the community [17]. It allows to simulate UHECR trajectories in structured magnetic fields that can be provided by the user. One example is shown in Fig. 4.

The nearby Universe is highly structured, both in terms of the distribution of UHECR sources and the cosmic magnetic fields in which UHECRs propagate. In Fig. 5 we show an example configuration based on work in Refs. [45, 46]. We note that the large scale distribution of cosmic magnetic is still rather uncertain. Future data from radio astronomy experiments such as Lofar and SKA will be very relevant to UHECR propagation studies since the will allow to map out these fields in much more detail. 

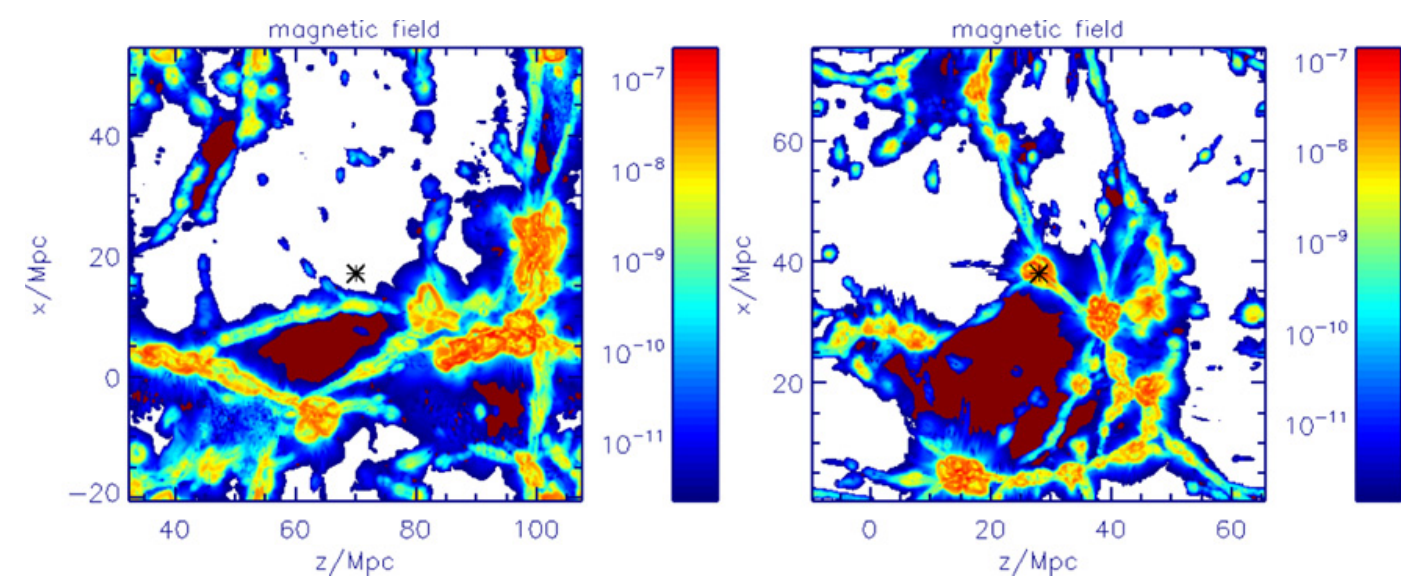

Figure 5. An observer (left panel) and a source position (right panel), marked by crosses, selected in the large scale magnetic field distributions from the simulations in Refs. $[45,46]$. The observer is in a "cold", largely unmagnetized environment, whereas the source is immersed in magnetic fields of $\sim 0.1 \mu \mathrm{G}$.

As an example we now investigate a situation in which a single discrete point source is situated at a distance of $\simeq 200 \mathrm{Mpc}$ from the observer injecting an $E^{-2}$ spectrum of pure protons up to $384.6 \mathrm{EeV}$. The results shown in the following are based on two 3D simulations in which a few $10^{10}$ particles were injected above $10^{18} \mathrm{eV}$ and above $3 \times 10^{19} \mathrm{eV}$, respectively, from one source per simulation box of volume of $(75 \mathrm{Mpc})^{3}$. In each such box which were periodically repeated one observer was placed an a detection was recorded if a particle crossed a sphere of radius $1 \mathrm{Mpc}$ around the observer. Overall about $10^{7}$ particles were detected, from which about $5 \times 10^{4}$ were from the one single source at $200 \mathrm{Mpc}$ considered. Figure 6 shows the delay time-energy distribution and the spectrum and sky image above $30 \mathrm{EeV}$ averaged over all times. Note that the delay time-energy distribution is not smooth but shows peaks and valleys. This is due to magnetic lensing in the highly structured magnetic fields: The neighborhood of certain trajectories are preferred and the associated flux shows an enhancement.

In Fig. 7 we fold the delay time-energy distribution with an activity time scale of $10^{5} \mathrm{yr}$ and show the resulting spectrum and sky image at a time of $2 \times 10^{6} \mathrm{yr}$ after the first photon would have arrived from this source. In contrast to the case of a continuously emitting source shown in Fig. 6, the spectrum is now a broad bump around $50 \mathrm{EeV}$. Lower energy particles have not arrived yet and high energy particles already passed due to their in general smaller time delay. Furthermore, the sky image is now much more concentrated around the source position and shows several images due to magnetic lensing.

This example shows that UHECR spectra and sky images of intermittent sources observed at a given time can be dramatically different from the ones for continuously emitting sources and also from how such sources appear in photons or potentially neutrinos. This has to be kept in mind when interpreting possible UHECR clusters in the sky such as the one suggested in Ref. [47].

Finally, the magnetic field of our own Galaxy plays a very important role in the interpretation of UHECR data. For heavy nuclei such as iron the deflection angles are expected to be of the order of $50^{\circ}$ up to the highest energies observed. At lower energies around $10^{18} \mathrm{eV}$ the propagation of lighter nuclei occurs at the transition between ballistic and diffusive propagation and has to be modelled with trajectory simulations. If the UHECR sources at this energy were galactic, this has important implications for the anisotropy of the flux of these UHECRs: Trajectory based simulations show [48] that a predominantly proton dominated composition would predict anisotropies in excess of the upper limits from the Pierre Auger experiment [49]. Only a heavy composition close to iron would predict anisotropies that are sufficiently small to be consistent with the upper limits due to their stronger deflection in the galactic magnetic fields. As a result, if the mass composition around $10^{18} \mathrm{eV}$ turns 

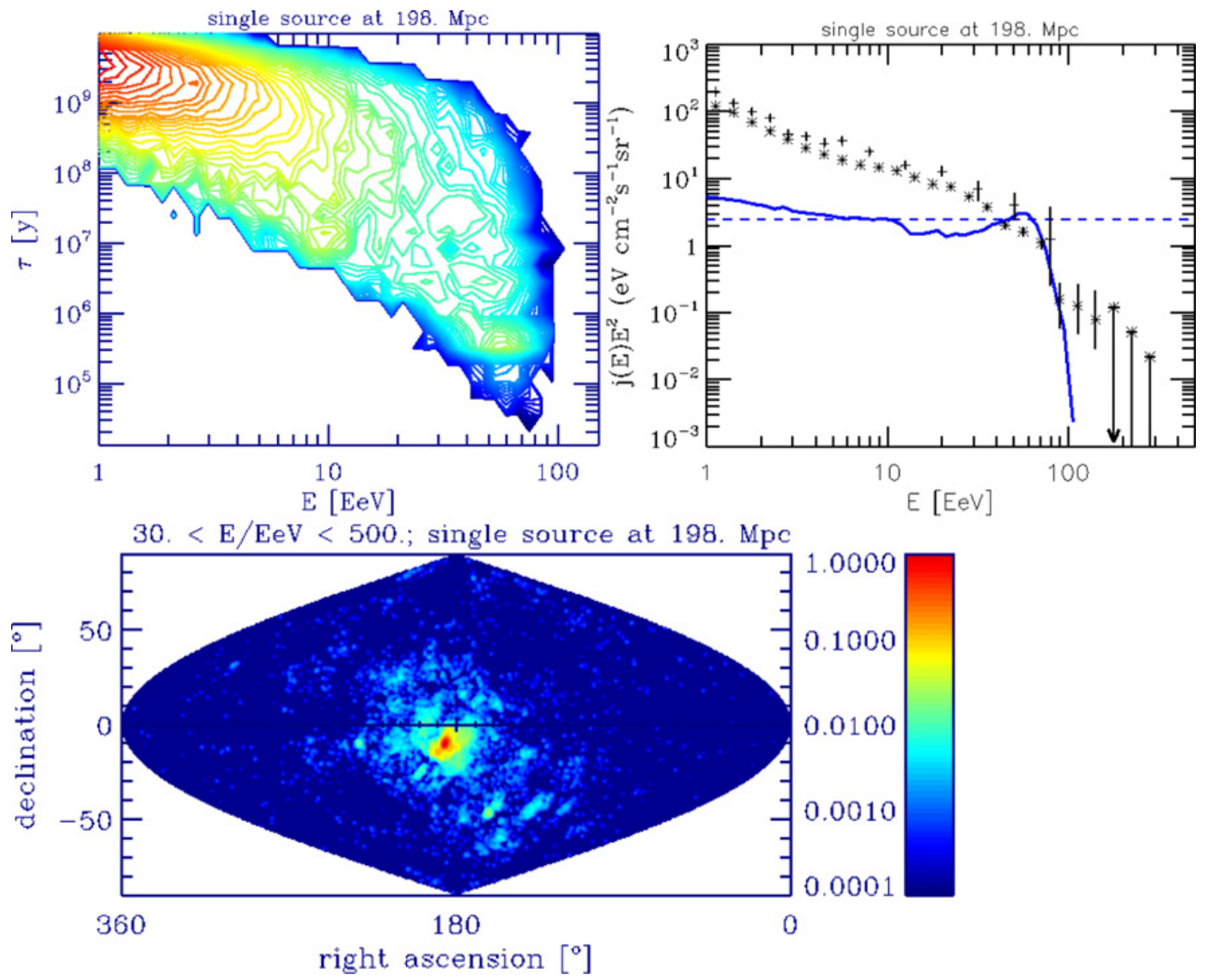

Figure 6. A pure proton source injects an $E^{-2}$ spectrum of pure protons up to $384.6 \mathrm{EeV}$ at a distance of $\simeq 200 \mathrm{Mpc}$ from the observer in the configuration shown in Fig. 5. Shown are the resulting delay time-energy distribution (upper panel), and the time-averaged spectrum (left lower panel) and sky image above $3 \times 10^{19} \mathrm{eV}$ (right lower panel).
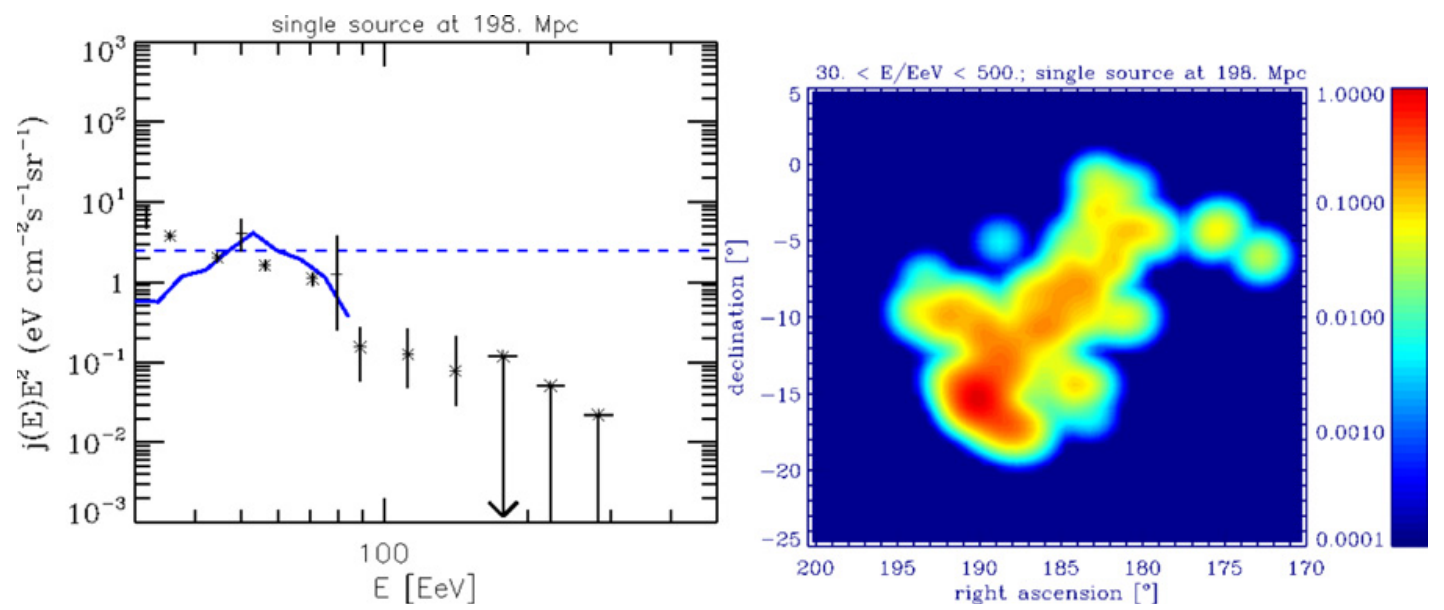

Figure 7. Spectrum (left panel) and sky image above $3 \times 10^{19} \mathrm{eV}$ (right panel) of the source at a time of $2 \times 10^{6}$ years after the source in the scenario from Fig. 5 and 6 would start to become visible in photons, i.e. for propagation without deflection, if the source would be active for $10^{5}$ years thereafter. 
out to be light, the UHECR flux at these energies would have to be dominantly extragalactic. This is potentially important information since somewhere between $\simeq 10^{17} \mathrm{eV}$ and the ankle at a few times $10^{18} \mathrm{eV}$ a cross-over from galactic to extragalactic cosmic rays is expected but the precise location in energy of this transition is currently still unknown.

\section{TESTING LORENTZ SYMMETRY WITH ULTRA-HIGH ENERGY COSMIC PARTICLES}

Both loop quantum gravity and string theory often break the Lorentz symmetry or realize it in ways different from the linear Lorentz transformation of special relativity. Such effects can manifest themselves through new terms in the dispersion relation, the relation between energy $E$ and momentum $p$ of a particle of mass $m$, that are suppressed by some power $n$ of the Planck mass $M_{\mathrm{Pl}} \simeq 1.22 \times$ $10^{19} \mathrm{GeV}$,

$$
E^{2}=m^{2}+p^{2}\left[1+\eta\left(\frac{p}{M_{\mathrm{Pl}}}\right)^{n}\right],
$$

where $\eta$ is a dimensionless number which should be of order unity if the corresponding term is allowed within the model. Such terms can modify both the propagation velocity of the particles and the kinematics of interactions.

Keeping only terms to first order in $m^{2}$ and $\eta$, the group velocity for Eq. (14) is given by

$$
v=\frac{\partial E}{\partial p} \simeq 1-\frac{m^{2}}{2 E^{2}}+\frac{\eta}{2}(n+1)\left(\frac{E}{M_{\mathrm{Pl}}}\right)^{n} \equiv 1-\frac{m^{2}}{2 E^{2}}+\delta(E),
$$

where $\delta(E) \equiv \eta(n+1)\left(E / M_{\mathrm{Pl}}\right)^{n} / 2$ is the deviation from the Lorentz-invariant velocity. For photons, $m=0$, this can lead to arrival time-delays between photons of different energies emitted by GRBs or by flares of active galactic nuclei. Based on observations of GRBs up to around $100 \mathrm{GeV}$, for $n=1$ one obtains limits of order unity, $|\eta| \lesssim 1[50]$.

The kinematics of interactions will be changed whenever the Lorentz invariance violating (LIV) terms become comparable to the mass $m_{l}$ of the lightest massive particle involved. This occurs for

$$
E \gtrsim E_{\mathrm{cr}}=\left(m_{l}^{2} M_{\mathrm{Pl}}^{n}\right)^{1 /(n+2)} .
$$

As a result, the larger the particle mass the higher the energy at which LIV effects come into play. Therefore, TeV electrons and positrons, but not protons, can be used to constrain $n=1 \mathrm{LIV}$ effects [51], and protons above $\sim 10^{18} \mathrm{eV}$ are required to obtain constraints on hadronic LIV terms with $n=2$ scaling. A specific case is pair production by photons for which $m_{l}=m_{e}$ is the electron mass. According to Eq. (14), pair production by a photon of energy $E_{\gamma}$ probes parameters of the size

$$
\eta \sim \frac{M_{\mathrm{Pl}}^{n} m_{e}^{2}}{E_{\gamma}^{n+2}} \sim\left\{\begin{array}{l}
10^{-14}\left(\frac{10^{18} \mathrm{eV}}{E_{\gamma}}\right)^{3} \text { for } n=1 \\
10^{-4}\left(\frac{10^{18} \mathrm{eV}}{E_{\gamma}}\right)^{4} \text { for } n=2
\end{array}\right.
$$

Furthermore, a positive $\eta$ would lead to spontaneous photon decay into an electron/positron pair above a certain energy threshold. A more detailed investigation [52, 53] shows that LIV parameters of the size in Eq. (17) can inhibit pair production if one assumes that energy and momentum are still conserved. This would lead to a very high UHE photon flux, comparable to the predicted UHE neutrino flux in the example of Fig. 3 which would be inconsistent with the photon flux upper limits from the Pierre Auger experiment [25]. As a result, certain combinations of LIV parameters of the size of Eq. (17) in the electron and photon sector can be strongly constrained already by the non-observation of UHE photons. The observation of a single UHE photon would extend these constraints such that all LIV terms 


\section{UHECR 2012}

in the dispersion relation of electrons and photons larger than $\simeq 10^{-14} p^{3} / M_{\mathrm{Pl}}$ would be ruled out. The corresponding small pre-factors would be unnatural which would imply that LIV terms suppressed to first and possibly even to second order in the Planck mass are probably absent altogether.

\section{CONCLUSIONS}

With a few selected examples we have shown how the interplay between high energy charged cosmic ray, $\gamma$-ray and neutrino fluxes can reveal important information on the nature of the sources and also the propagation of these particles. With more data expected this will become more and more important in the future. Furthermore, multi-messenger studies will include gravitational waves which are expected to be detected within a few years and will provide detailed and complementary information on violent astrophysical events such as supernova explosions and the mergers of supermassive black holes.

This work was supported by the Deutsche Forschungsgemeinschaft through the collaborative research centre SFB 676, by BMBF under grant 05A11GU1, and by the "Helmholtz Alliance for Astroparticle Physics (HAP)" funded by the Initiative and Networking Fund of the Helmholtz Association. The author also acknowledges support from the State of Hamburg, through the Collaborative Research program "Connecting Particles with the Cosmos".

\section{References}

[1] R. Blandford, D. Eichler, Phys. Rept. 154, 1-75 (1987)

[2] D. B. Melrose, arXiv:0902.1803 [astro-ph.SR]

[3] A. M. Hillas, Ann. Rev. Astron. Astrophys. 22 (1984) 425

[4] R. V. E. Lovelace, Nature 262, 649 (1976)

[5] R. D. Blandford, Phys. Scripta T85, 191 (2000)[arXiv:astro-ph/9906026]

[6] K. Ptitsyna and S. Troitsky, [arXiv:0808.0367 [astro-ph]]

[7] M. V. Medvedev, Phys. Rev. E 67, 045401 (2003) [arXiv:astro-ph/0303271]

[8] P. Abreu et al. [Pierre Auger Collaboration], Astropart. Phys. 34, 314 (2010) [arXiv:1009.1855 [astro-ph.HE]].

[9] Fermi LAT Collaboration, Science 328, 725 (2010) [arXiv:1006.3986 [astro-ph.HE]].

[10] A. Falcone, H. Hase, C. Pagoni and C. Ploetz [Fermi Collaboration], Astrophys. J. 719, 1433 (2010) [arXiv:1006.5463 [astro-ph.HE]].

[11] F. Aharonian et al., Astrophys. J. Lett. 695, L40 (2009)

[12] S. Sahu, B. Zhang and N. Fraija, Phys. Rev. D 85, 043012 (2012) [arXiv:1201.4191 [astroph.HE]].

[13] see, e.g., F. M. Rieger, F. A. Aharonian, [arXiv:0910.2327 [astro-ph.HE]].

[14] M. Kachelriess, S. Ostapchenko and R. Tomas, New J. Phys. 11, 065017 (2009) [arXiv:0805.2608 [astro-ph]]; M. Kachelriess, S. Ostapchenko and R. Tomas, arXiv:1002.4874 [astro-ph.HE]

[15] A. Kusenko and M. B. Voloshin, Phys. Lett. B 707, 255 (2012) [arXiv:1109.0565 [astro-ph.HE]]

[16] M. J. Hardcastle, arXiv:1003.2500 [astro-ph.HE]

[17] K.-H. Kampert, J. Kulbartz, L. Maccione, N. Nierstenhoefer, P. Schiffer, G. Sigl and A. R. van Vliet, arXiv:1206.3132 [astro-ph.IM]

[18] M. A. DuVernois and M. R. Thayer, "The elemental composition of the galactic cosmic-ray source: ULYSSES high-energy telescope results,” Astrophys. J. 465, 982 (1996)

[19] D. Allard, E. Parizot, E. Khan, S. Goriely and A. V. Olinto, "UHE nuclei propagation and the interpretation of the ankle in the cosmic-ray spectrum," Astron. Astrophys. 443, L29 (2005) [arXiv:astro-ph/0505566] 
[20] J. Abraham et al. [Pierre Auger Collaboration], Phys. Lett. B 685, 239 (2010) [arXiv:1002.1975 [astro-ph.HE]]

[21] P. Abreu et al. [Pierre Auger Collaboration], arXiv:1107.4809 [astro-ph.HE]

[22] R. Abbasi et al. [IceCube Collaboration], Phys. Rev. D 83, 092003 (2011) [Erratum-ibid. D 84, 079902 (2011)] [arXiv:1103.4250 [astro-ph.CO]]

[23] P. Abreu et al. [Pierre Auger Collaboration], Phys. Rev. D 84, 122005 (2011) [arXiv:1202.1493 [astro-ph.HE]]

[24] P. W. Gorham et al. [ANITA Collaboration], Phys. Rev. D 85, 049901 (2012) [arXiv:1011.5004 [astro-ph.HE], arXiv:1003.2961 [astro-ph.HE]]

[25] J. Abraham et al. [The Pierre Auger Collaboration], Astropart. Phys. 31, 399 (2009) [arXiv:0903.1127 [astro-ph.HE]]

[26] A. A. Abdo et al. [Fermi-LAT Collaboration], Phys. Rev. Lett. 104, 101101 (2010) [arXiv:1002.3603 [astro-ph.HE]]

[27] see, e.g., K. Kotera and A. V. Olinto, Ann. Rev. Astron. Astrophys. 49, 119 (2011). arXiv:1101.4256 [astro-ph.HE]

[28] D. Horns and M. Meyer, JCAP 1202, 033 (2012) [arXiv:1201.4711 [astro-ph.CO]]

[29] W. Essey, O. Kalashev, A. Kusenko and J. F. Beacom, Astrophys. J. 731, 51 (2011) [arXiv:1011.6340 [astro-ph.HE]]

[30] A. Neronov, I. Vovk, Science 328, 73 (2010). [arXiv:1006.3504 [astro-ph.HE]]

[31] W. Essey, S. 'i. Ando and A. Kusenko, Astropart. Phys. 35, 135 (2011) [arXiv:1012.5313 [astroph.HE]]

[32] R. Abbasi et al. [IceCube Collaboration], Astrophys. J. 710, 346 (2010) [arXiv:0907.2227 [astroph.HE]]

[33] R. Abbasi et al. [ IceCube Collaboration ], Phys. Rev. Lett. 106, 141101 (2011). [arXiv:1101.1448 [astro-ph.HE]]

[34] R. Abbasi et al. [IceCube Collaboration], Nature 484, 351 (2012) [arXiv:1204.4219 [astroph.HE]]

[35] A. Ishihara et al. [IceCube Collaboration], talk given at XXV International Conf. on Neutrino Physics and Astrophysics 2012, Kyoto, Japan

[36] E. Waxman, Phys. Rev. Lett. 75, 386-389 (1995) [astro-ph/9505082]

[37] see, e.g., C. D. Dermer, arXiv:1008.0854 [astro-ph.HE]

[38] M. Ahlers, M. C. Gonzalez-Garcia, F. Halzen, Astropart. Phys. 35, 87-94 (2011). [arXiv:1103.3421 [astro-ph.HE]].

[39] Z. Li, Phys. Rev. D 85, 027301 (2012) [arXiv:1112.2240 [astro-ph.HE]]

[40] W. Winter, arXiv:1201.5462 [astro-ph.HE]

[41] P. Baerwald, S. Hummer and W. Winter, Astropart. Phys. 35, 508 (2012) [arXiv:1107.5583 [astroph.HE]]

[42] S. Hummer, P. Baerwald and W. Winter, Phys. Rev. Lett. 108, 231101 (2012) [arXiv:1112.1076 [astro-ph.HE]]

[43] G. R. Farrar and A. Gruzinov, Astrophys. J. 693, 329 (2009) [arXiv:0802.1074 [astro-ph]]

[44] G. Sigl, New J. Phys. 11, 065014 (2009)

[45] F. Miniati,"Inter-galactic Shock Acceleration and the Cosmic Gamma-ray Background," Mon. Not. Roy. Astron. Soc. 337, 199 (2002) [arXiv:astro-ph/0203014]

[46] G. Sigl, F. Miniati and T. A. Enßlin, "Ultra-high energy cosmic ray probes of large scale structure and magnetic fields," Phys. Rev. D 70, 043007 (2004) [arXiv:astro-ph/0401084]

[47] G. R. Farrar, A. A. Berlind and D. W. Hogg, Astrophys. J. 642, L89 (2006) [astro-ph/0507657]

[48] G. Giacinti, M. Kachelriess, D. V. Semikoz and G. Sigl, (to appear in JCAP) [arXiv:1112.5599 [astro-ph.HE]] 


\section{UHECR 2012}

[49] P. Abreu et al. [The Pierre Auger Collaboration], Astropart. Phys. 34, 627 (2011) [arXiv:1103.2721 [astro-ph.HE]]

[50] A. A. Abdo et al. Nature 462 (2009) 331

[51] see, e.g., L. Maccione, S. Liberati, A. Celotti et al., JCAP 0710, 013 (2007). [arXiv:0707.2673 [astro-ph]]

[52] M. Galaverni and G. Sigl, Phys. Rev. Lett. 100, 021102 (2008) [arXiv:0708.1737 [astro-ph]]

[53] M. Galaverni and G. Sigl, Phys. Rev. D 78, 063003 (2008) [arXiv:0807.1210 [astro-ph]] 